\title{
EDYTA KOCIUBIŃSKA
}

Université Catholique de Lublin JP II

Contre, tout contre.

Le manifeste révolutionnaire du dandy

n définissant l'esprit de révolte du dandy, per-
sonnage phare de la vie mondaine au XIX siècle, Jules Barbey d'Aurevilly indique que le dandysme " se joue de la règle et pourtant la respecte encore. II en souffre et s'en venge tout en la subissant; il s'en réclame quand il y échappe ; il la domine et en est dominé tour à tour ${ }^{1}$. Tout en essayant de changer sa vie en œuvre d'art, professant " le culte de la différence dans le siècle de l'uniforme $\aleph^{2}$, le dandy manifestera son désaccord envers les lois qui l'emprisonnent et lui imposent des règles étriquées. En cultivant l'idée du beau dans sa personne, il élève une protestation désespérée contre la décadence et la corruption, contre la sottise humaine et contre la vulgarité.

Comme le dandy " ne peut se poser qu'en s'opposant ${ }^{3}$, notre article tentera de dresser la liste des postulats de cet individu rebelle, qui est " contre, tout contre " pour reprendre la célèbre formule de Sacha Guitry. Nous tenterons de créer une sorte de manifeste du dandy, " un révolutionnaire sans révolution ${ }^{4}$, un élégant et charmant

$1 \mathrm{~J}$. Barbey d'Aurevilly, Du Dandysme et de George Brummell, Paris, Les Éditions de Paris, 2008, p. 32.

${ }^{2}$ R. Kempf, Dandies. Baudelaire et Cie, Paris, Seuil, 1977, p. 9.

${ }^{3}$ A. Camus, L'Homme révolté, Paris, Gallimard, 1985, p. 71.

4 Ph. Sollers, "Métaphysique du dandysme ", [dans : ] Le Nouvel Observateur, le 22 décembre 2011, n 2459-2460, www.philippesollers.net/ metaphysique-du-dandysme.html. 
contestataire. Nous nous inspirerons des textes des plus grands théoriciens du dandysme au XIX siècle, à savoir Jules Barbey d'Aurevilly, Charles Baudelaire, Jules Lemaître et Oscar Wilde.

\section{Contre le mauvais goût}

Considérant l'esthétique en tant que morale suprême, le dandy s'adonne au culte du beau, il va jusqu'à partir à la recherche de la beauté perdue. Il est dégoûté par la société plongée dans la laideur, voire écrasée par le mauvais goût de la bourgeoisie qui agit comme un venin empoisonné. En tant qu'esthète, il choisit l'art et l'amour de soi comme les seules et uniques valeurs auxquelles il veuille bien être fidèle, il rejette donc consciemment les revendications de l'éthique à gouverner son existence. D'après Oscar Wilde " [I] dandysme est l'affirmation de la modernité absolue de la Beauté $\iota^{5}$, par contre la beauté - continue-t-il - « est une forme de génie, car elle ne requiert aucune explication $»^{6}$.

Comme le note Valérie d'Alkemade, c'est grâce à l'art que le dandy, essayant constamment de lutter contre l'animalité originelle de l'homme, réussit à accéder à son moi idéal. Par là même, son apparence extérieure ainsi que son habit deviennent les instruments par lesquels va s'affirmer la préoccupation esthétique. Le dandy " va se donner à voir comme une œuvre d'art incarnée, son corps faisant fonction de laboratoire expérimental dans sa tentative de plus en plus hardie de domestication de l'animalité " ${ }^{7}$. Toutes les expériences visant à faire de son existence une œuvre d'art vivante, selon l'aphorisme de Wilde, sont aussi des preuves de résistance, de désaccord

\footnotetext{
5 O. Wilde, Quelques maximes pour l'instruction des personnes trop instruites, [dans :] Idem, CEuvres, Paris, Gallimard, 1996, p. 968.

6 O. Wilde, Le Portrait de Dorian Gray, [dans :] Idem, CEuvres, op. cit., p. 369.

${ }^{7}$ V. d'Alkemade, Dandys. Abécédaire impertinent du dandysme et des néodandys, Bruxelles, Soliflor, 2007, p. 36.
} 
envers le monde qui veut l'engloutir. On peut avancer I'hypothèse que son opposition donne naissance à une sorte $d^{\prime}$ " élite artiste ${ }^{8}$ selon l'expression de Nathalie Heinich, une caste qui réussit à préserver le culte de l'originalité dans ce monde hostile à la créativité. Philippe Sollers trouve que le dandy est suprêmement " grec ", au sens de Nietzsche parlant des Grecs de l'Antiquité qui " s'arrêtent vaillamment à la surface, croient à tout l'Olympe de l'apparence, sont superficiels par profondeur ${ }^{9}$. La référence à Nietzsche est remarquablement analysée par Daniel Salvatore Schiffer qui compare la figure du dandy au philosophe-artiste, cet être pour qui la vie se doit d'être une œuvre d'art ${ }^{10}$.

\section{Contre la vulgarité et la médiocrité}

Le dandy est " l'antithèse radicale de l'homme des foules ${ }^{11}$, selon l'expression de Michel Onfray. Sa tâche première est d'éveiller la curiosité, de provoquer la société menacée par la médiocrité, proie facile des goûts vulgaires. Baudelaire dira que tous les dandys " participent du même caractère d'opposition et de révolte ; tous sont des représentants de ce qu'il y a de meilleur dans l'orgueil humain, de ce besoin, trop rare chez ceux d'aujourd'hui, de combattre et de détruire la trivialité ${ }^{12}$. En cherchant un moyen efficace qui permettra de sauver l'humanité de la catastrophe, il ne voit qu'une solution : " s'offrir en spectacle, [...] laisser au public le droit d'être fasciné par l'énigme vivante qu'il représente ${ }^{13}$. Autour de lui

\footnotetext{
${ }^{8} \mathrm{~N}$. Heinich, L'Élite artiste. Excellence et singularité en régime démocratique, Paris, Gallimard, 2015, p. 241.

${ }^{9} \mathrm{Ph}$. Sollers, "Métaphysique du dandysme ", op. cit.

${ }^{10} \mathrm{D}$. Salvatore Schiffer, Philosophie du dandysme. Une esthétique de l'âme et du corps, Paris, PUF, 2008, p. 123-128.

$11 \mathrm{M}$. Onfray, Politique de rebelle. Traité de résistance et d'insoumission, Paris, Le Livre de Poche, 1999, p. 227.

$12 \mathrm{Ch}$. Baudelaire, Le Peintre de la vie moderne, [dans :] Idem, CEuvres complètes, Y.-G. Le Dantec (éd. critique), Paris, Gallimard, 1954, p. 908.

${ }^{13} \mathrm{M}$. Foerster, L'Art d'être odieux. Nouveaux essais sur le dandysme, Paris,
} 
circulent des individus privés d'inquiétudes, contents d'eux-mêmes et de la vie médiocre mais tranquille qu'ils mènent : il en est déçu, et il se défend d'être condamné à la même existence morne. Le dégoût le pousse à se refuser à végéter comme eux, à accepter la banalité et l'envahissement de la monotonie.

Quelle est donc son arme privilégiée ? II s'agit bel et bien du raffinement, qui unit la perfection et l'originalité. Rappelons le premier dandy, inimitable George Brummell, qui se voulait raffiné, mais sans se distinguer réellement. Sa révolte individuelle s'exprimait dans son habillement par une grande élégance et une légère originalité, tout juste perceptible. Pour le dandy, "épris avant tout de distinction ", la perfection de la toilette consiste dans " la simplicité absolue, qui est en effet la meilleure manière de se distinguer ${ }^{14}$. C'est cette sobriété de la mise qui deviendra le signe de ralliement de tous ceux qui ont décidé de défendre le sublime contre la médiocrité envahissante. Honoré de Balzac constatera que " c'est moins la simplicité de luxe, qu'un luxe de simplicité ${ }^{15}$. Grâce à son allure soignée, le dandy n'est plus une figure anonyme, bien au contraire, la société le perçoit comme un individu hors du commun. Comme le montre avec finesse Jules Lemaître, il réussira à faire croire que " d'innover en fait d'usages mondains, de conversations élégantes, d'habits, de manières et d'amusements, c'est aussi rare, aussi méritoire, aussi digne de considération que d'inventer et de créer en politique, en art, en littérature ${ }^{16}$. Ainsi, le dandy

Jean Paul Bayol, 2010, p. 15. Charles Baudelaire ajoutera que le dandy " ne peut jamais être un homme vulgaire. S'il commettait un crime, il ne serait pas déchu peut-être ; mais si ce crime naissait d'une source triviale, le déshonneur serait irréparable "(Ch. Baudelaire, Le Peintre de la vie moderne, op. cit., p. 907).

${ }^{14} \mathrm{Ch}$. Baudelaire, Le Peintre de la vie moderne, op. cit., p. 907.

$15 \mathrm{H}$. de Balzac, Traité de la vie élégante, suivi de la Théorie de la démarche, C. Varèze (éd. critique), Paris, Bossard, 1922, p. 109.

$16 \mathrm{~J}$. Lemaître, Les Contemporains, $4^{e}$ série, (2e édition), Paris, H. Lecène et H. Houdin, 1889, p. 56-58. 
se crée un monde à part où il règne à l'aide de son code de l'élégance sublime et des manières recherchées comme les autres règnent grâce à l'intelligence, la puissance ou la fortune.

\section{Contre l'utilité}

Un autre postulat important est celui de la révolte contre le piège de l'utilité qui ôte aux dandys la gratuité de leur geste. Dans ce siècle qui professe le culte du labeur, le dandy se prononce pour le culte " de l'inutile et de l'artifice, du loisir et de la gratuité là où la plupart s'épuisent dans l'utile, le travail, la rente ${ }^{17}$. L'une des principales lois non-écrites du dandysme avertit ses partisans qu'il faut rester fidèle à la pratique du désœuvrement aristocratique, donc qu'il est défendu de s'abaisser au travail. La seule idée de faire carrière, d'être un arriviste le dégoûte. Qui plus est, il dédaigne l'argent, le considérant uniquement comme un moyen de réaliser ses projets. Comme le souligne Baudelaire, la fortune est " indispensable aux gens qui se font un culte de leurs passions ", mais à vrai dire, " le dandy n'aspire pas à l'argent comme à une chose essentielle ; un crédit illimité pourrait lui suffire ${ }^{18}$. En effet, il s'oppose à ce que l'argent limite sa liberté.

$S^{\prime}$ il est tout de même contraint au labeur, il s'y résout avec le plus grand désintéressement. "Être un homme utile m'a toujours paru quelque chose de bien hideux ${ }^{19}$, annonce ouvertement Baudelaire en expliquant que les êtres d'exception sont en quelque sorte prédestinés à l'oisiveté, preuve de luxe et d'excès. Or, selon la morale moderne, le culte de l'inutilité constitue une violation des règles sociales, les êtres improductifs devenant des bannis, des hors-la-loi indignes de respect. Le dandy se décide

\footnotetext{
$17 \mathrm{M}$. Onfray, Politique de rebelle. Traité de résistance et d'insoumission, op. cit., p. 226.

${ }^{18} \mathrm{Ch}$. Baudelaire, Le Peintre de la vie moderne, op. cit., p. 907.

${ }^{19} \mathrm{Ch}$. Baudelaire, Mon cœur mis à nu, [dans :] Idem, Euvres complètes, op. cit., p. 1209.
} 
à lutter contre ce stéréotype, il n'accepte pas d'être dépendant d'une occupation qui assure l'estime ou garantit les faux honneurs. Toujours à rebours de son siècle, il refuse de s'humilier en devenant l'esclave de quelqu'un.

\section{Contre les règles, les convenances}

Le dandy se prend pour la première et l'ultime source des valeurs auxquelles il est fidèle et qu'il est libre de modifier selon ses caprices. II n'est pas obligé de prouver à chaque pas son individualisme, voire égotisme : il refuse de se soumettre aux règles imposées en réalisant " le dernier éclat d'héroïsme dans les décadences $\$ 20$ dont parle Baudelaire. Rappelons que Barbey d'Aurevilly, dans son essai sur George Brummell, nous promet la codification des règles dandys pour finalement se libérer de cette contrainte quelques pages après, en annonçant que « [c]e qui fait le Dandy, c'est l'indépendance. Autrement, il y aurait une législation du Dandysme, et il n'y en a pas ${ }^{21}$.

Or, le dandy maîtrise parfaitement tous les codes du jeu social en vigueur, il s'en sert pour mieux les mépriser et en profite pour créer les règles de son propre jeu. II doit juste vérifier jusqu'à quel moment on peut jouer avec le feu, sans tout de même dépasser trop les limites et s'attirer les foudres de la société. Comme le signale Émilien Carassus, le dandy procède à un stratagème de provocation, il veut être remarqué, mais être remarqué à l'intérieur d'un groupe qu'il domine. Si on le rejette, s'il devient un paria provoquant la désapprobation générale, il ne lui reste qu'à accepter sa chute ${ }^{22}$. Et pourtant, on attend avec impatience et angoisse ses décrets et ses extravagances. On espère qu'il surprendra, on le critique, mais on l'imite en même temps. Ses discours, ses gestes, ses spectacles

${ }^{20} \mathrm{Ch}$. Baudelaire, Le Peintre de la vie moderne, op. cit., p. 907.

$21 \mathrm{~J}$. Barbey d'Aurevilly, Du dandysme et de George Brummell, op. cit., p. 50.

${ }^{22}$ Cf. É. Carassus, Le Mythe du dandy, Paris, Armand Colin, 1971, p. 113. 
ainsi que ses astuces séduisantes font la preuve de son talent d'illusionniste. II incarne à merveille " une belle figure antimoderne : l'individualiste réfractaire et rebelle $»^{23}$.

\section{Contre la nature}

Le dandy décide de s'affranchir non seulement des lois que lui dicte la société, mais aussi de celles imposées par la nature. Pourquoi nourrit-il un sentiment de haine envers la nature, pourquoi en vient-il même à la rejeter ? S'opposant au mythe du "bon sauvage " qui prône que tout ce qui est naturel est beau, Baudelaire proteste : " Passez en revue, analysez tout ce qui est naturel, [...] vous ne trouverez rien que d'affreux. Tout ce qui est beau et noble est le résultat de la raison et du calcul ${ }^{24}$.

En se prononçant en faveur de subterfuges, le dandy ne perçoit plus la nature en tant que refuge, consolation ou source de passions. Au contraire, tout ce qui est naturel le répugne, car la nature est vulgaire, elle tire l'homme vers l'animal en éveillant ses instincts les plus abominables. Qui plus est, " [a]ccepter la nature, vivre naturellement, c'est-à-dire en accord avec les lois physiques, biologiques, sociales et morales, serait accepter de noyer l'étincelle que porte l'artiste, dans l'ennui, dans la médiocrité et la laideur humaines et sociales ${ }^{25}$. Le dandy, ce " feu latent " selon l'expression baudelairienne, est persuadé qu'il peut se passer de la nature, ou du moins, la corriger à son gré : "[j]e voudrais ", avoue le poète des Fleurs du Mal, "les prairies teintées en rouge, les rivières jaune d'or et les arbres peints en bleu. La nature n'a pas

\footnotetext{
${ }^{23}$ A. Compagnon, Les Antimodernes. De Joseph de Maistre à Roland Barthes, Paris, Gallimard, 2005, p. 129. Cf. aussi A. I. François, E. Kociubińska, G. Pham-Thanh, P. Zoberman (dirs.), Figures du dandysme, coll. Études de littérature, linguistique et art, Frankfurt am Main, Peter Lang, 2017, v. 25.

${ }^{24}$ Ch. Baudelaire, Le Peintre de la vie moderne, op. cit., p. 919.

${ }^{25}$ M. Lemaire, Le Dandysme de Baudelaire à Mallarmé, Montréal, Presses de l’Université de Montréal, 1978, p. 37.
} 
d'imagination ${ }^{26}$. Ainsi, le dandy choisit l'artifice qui lui permet de rivaliser avec la nature. Il s'acharnera à I'humilier pour pouvoir ensuite triompher d'elle en instaurant un vrai culte de l'autocréation. Comme le note Jean-Pierre Saïdah, " art et artifice sont ici intimement liés, afin de réaliser le vœu ultime du dandy : créer et se créer ${ }^{27}$.

\section{Contre les sentiments}

La fameuse devise de Barbey d'Aurevilly définit l'obligation première de chaque dandy : " produire la surprise en gardant l'impassibilité ${ }^{28}$ et, renchérit Baudelaire, " le plaisir d'étonner et la satisfaction orgueilleuse de ne jamais être étonné ${ }^{29}$. Cette insistance sur le contrôle permanent des sentiments auxquels est condamné le dandy explique bien pourquoi l'impassibilité est l'unique vertu à laquelle il doit être fidèle. Afin de jouer avec les sentiments des autres, il se plaît à changer les visages tout en restant imperturbable pour se moquer de ses semblables. Comme le constate, avec une franchise déconcertante, Oscar Wilde, "[à] une époque aussi vulgaire que la nôtre, nous avons tous besoin de masques ${ }^{30}$.

Protégé par un masque, le dandy déroute son public car au moment où ce dernier semble désarmé par son impassibilité inébranlable, il s'en sert pour attaquer son adversaire. II se rend compte que les sentiments témoignent d'une faiblesse, que chaque émotion détruit le plan machiavélique à l'aide duquel il manipule ses vic-

${ }^{26}$ Anecdote rapportée par Jules Levallois, secrétaire de Sainte-Beuve, et citée par G. Sagnes, L'Ennui dans la littérature française de Flaubert à Laforgue, Paris, A. Colin, 1969, p. 325.

27 J.-P. Saïdah, "Artifice ", [dans :] A. Montandon (dir.), Dictionnaire du dandysme, Paris, Honoré Champion, 2016, p. 59.

28 J. Barbey d'Aurevilly, Du Dandysme et de George Brummell, op. cit., p. 28.

${ }^{29} \mathrm{Ch}$. Baudelaire, Le Peintre de la vie moderne, op. cit., p. 907.

30 O. Wilde, Lettre à Ph. Houghton, février 1894, [dans :] Idem, La Ballade de la geôle de Reading/The Ballad of the Reading Gaol, J. Besson (trad. et éd. critique), Paris, L'Âge d'Homme, 1989, p. 87. 
times. S'émouvoir équivaut à admettre que l'on est un ange déchu, un faible mortel condamné à souffrir comme les autres. Les sensations qui dominent chaque décision de l'homme le privent de tout contrôle. Conscient de toutes ces menaces, égoïste et égotiste, le dandy renonce aux passions, surtout à la plus dangereuse d'entre elles, l'amour, car " aimer, même dans le sens le moins élevé du mot, désirer, c'est toujours dépendre, c'est être esclave de son désir ${ }^{31}$. Une femme pourrait le ramener à la manifestation d'impulsions incontrôlées, elle détruirait à coup sûr la froide domination dont il se vante. Par fidélité à son impassibilité, par son masque impénétrable, il est un homme qui ne dévoile pas ses émotions et préfère rester une énigme de marbre, pareil au Sphinx.

\section{Conclusion}

En fait, comme le note avec pertinence Roger Kempf, "le dandy récuse dogmes et injonctions, opposant le singulier au multiple, le peu au trop, les vacances au labeur, la gratuité au profit, la richesse à l'enrichissement, la réserve à l'effusion et le délire de sa rigueur à la morne économie des ménages ${ }^{32}$. Il ne s'agit pas d'une simple pose, mais d'une vraie philosophie de l'existence à laquelle le dandy voue un culte sans bornes. C'est un révolté qui trouve qu'il n'y a de gouvernement raisonnable et assuré que l'aristocratique. II s'oppose à la monarchie ou la république basées sur la démocratie, car cette dernière repose sur l'idée que tous les hommes sont égaux, donc pareils, alors que ce fait est inadmissible pour le dandy qui est fier de sa singularité et son non-conformisme. La démocratie pour lui est la victoire de la médiocrité uniforme qui va tout emporter et tout submerger, comme l'explique très clairement Baudelaire dans son Peintre de la vie moderne:

31 J. Barbey d'Aurevilly, Du Dandysme et de George Brummell, op. cit., p. 46.

32 R. Kempf, Dandies. Baudelaire et Cie, op. cit., p. 10. 
«Mais, hélas ! la marée montante de la démocratie, qui envahit tout et qui nivelle tout, noie jour à jour ces derniers représentants de l'orgueil humain et verse des flots d'oubli sur les traces de ces prodigieux myrmidons ${ }^{33}$.

Comment donc définir cette révolte impassible du dandy ? De soi(e), de velours, par le sublime, comme le suggère Daniel Salvatore Schiffer ? $^{34}$ Incontestablement, toutes ces appellations rendent bien le caractère de cette insurrection, et nous pourrions en ajouter une autre : une révolution héroïco-utopique. Elle reste le symbole de la liberté de création, de la beauté gratuite, bien qu'elle soit dès le début condamnée à l'échec vu le caractère éphémère du dandysme, qui s'éteint lentement englouti par la démocratisation de la société. Toujours solitaire, le dandy mène sa vie d'artiste, d'adorateur de beauté suprême dans un respect sans bornes. En tant qu'idole crépusculaire, il " incarne pour son temps la figure de proue insensible aux tempêtes, et trace un style de vie servant d'exemple, son orgueilleux chemin vers I'horizon de sa mort, indifférent aux dires et aux faires de qui se targue de le suivre ${ }^{35}$. Conscient qu'il représente une espèce condamnée à l'extinction, il se retire du théâtre de la vie mondaine, mais jusqu'au bout il reste fidèle aux postulats de son manifeste, en prouvant avec fierté son indépendance.

Date de réception de l'article : 16.03.2018. Date d'acceptation de l'article : 30.03.2018.

${ }^{33} \mathrm{Ch}$. Baudelaire, Le Peintre de la vie moderne, op. cit., p. 908.

${ }^{34}$ Cf. D. Salvatore Schiffer, Le Dandysme, dernier éclat d'héroïsme, Paris, PUF, 2010 ; du même auteur : Le Dandysme : la création de soi, Paris, François Bourin Éditeur, 2011.

35 F. Dolto, Dandy, solitaire et singulier, Paris, Mercure de France, 1999, p. 21. 


\section{bibliographie}

Alkemade V. de., Dandys. Abécédaire impertinent du dandysme et des néo-dandys, Bruxelles, Soliflor, 2007.

Balzac H. de., Traité de la vie élégante, suivi de la Théorie de la démarche, C. Varèze (éd. critique), Paris, Bossard, 1922.

Barbey d'Aurevilly J., Du Dandysme et de George Brummell, Paris, Les Éditions de Paris, 2008.

Baudelaire Ch., Le Peintre de la vie moderne, [dans :] Idem, CEuvres complètes, Y.-G. Le Dantec (éd. critique), Paris, Gallimard, 1954.

Baudelaire Ch., Mon cœur mis à nu, [dans :] Idem, CEuvres complètes, Y.-G. Le Dantec (éd. critique), Paris, Gallimard, 1954.

Camus A., L'Homme révolté, Paris, Gallimard, 1985.

Carassus É., Le Mythe du dandy, Paris, Armand Colin, 1971.

Compagnon A., Les Antimodernes. De Joseph de Maistre à Roland Barthes, Paris, Gallimard, 2005.

Dolto F., Dandy, solitaire et singulier, Paris, Mercure de France, 1999.

Foerster M., L'Art d'être odieux. Nouveaux essais sur le dandysme, Paris, Jean Paul Bayol, 2010.

François A. I., Kociubińska E., Pham-Thanh G., Zoberman P. (dirs.), Figures du dandysme, coll. Études de littérature, linguistique et art, Frankfurt am Main, Peter Lang, 2017, v. 25.

Heinich N., L'Élite artiste. Excellence et singularité en régime démocratique, Paris, Gallimard, 2015.

Kempf R., Dandies. Baudelaire et Cie, Paris, Seuil, 1977.

Kociubińska E., L'insoutenable pesanteur de l'être. Le dandysme en France au XIXe siècle et son rayonnement en Europe, Lublin, Wydawnictwo KUL, 2015.

Lemaire M., Le Dandysme de Baudelaire à Mallarmé, Montréal, Presses de l'Université de Montréal, 1978.

Lemaître J., Les Contemporains, $4^{e}$ série, (2e édition), Paris, $\mathrm{H}$. Lecène et $\mathrm{H}$. Houdin, 1889.

Onfray M., Politique de rebelle. Traité de résistance et d'insoumission, Paris, Le Livre de Poche, 1999.

Sagnes G., L'Ennui dans la littérature française de Flaubert à Laforgue, Paris, A. Colin, 1969.

Saïdah J.-P., "Artifice ", [dans :] A. Montandon (dir.), Dictionnaire du dandysme, Paris, Honoré Champion, 2016.

Salvatore Schiffer D., Philosophie du dandysme. Une esthétique de l'âme et $d u$ corps, Paris, PUF, 2008.

Salvatore Schiffer D., Le Dandysme, dernier éclat d'héroïsme, Paris, PUF, 2010.

Salvatore Schiffer D., Le Dandysme : la création de soi, Paris, François Bourin Éditeur, 2011.

Sollers Ph., "Métaphysique du dandysme ", [dans :] Le Nouvel Observateur, le 22 décembre 2011, n 2459-2460, www.philippesollers.net/ metaphysique-du-dandysme.html.

Wilde O., La Ballade de la geôle de Reading/The Ballad of the Reading Gaol, J. Besson (trad. et éd. critique), Paris, L'Âge d'Homme, 1989. 
Wilde O., Le Portrait de Dorian Gray, [dans :] Idem, CEuvres, Paris, Gallimard, 1996.

Wilde O., Quelques maximes pour l'instruction des personnes trop instruites, [dans :] Idem, CEuvres, Paris, Gallimard, 1996.

\section{abstract}

\section{Against All Odds. A Revolutionary Dandy Manifesto}

The paper aims to show a dandy as a performer who acts as if he was guided by a kind of a revolutionary manifesto, which we attempt to recreate by referring to essays of Jules Barbey d'Aurevilly, Charles Baudelaire, Jules Lemaitre or Oscar Wilde. The dandy's rebellion against the outside world consists in the creation of his own personality, as well as in the rejection of the rudimentary uniformity and the duty of utility. He seeks to give his life an exceptional character, his unique goal is to serve the highest value: the Beauty. His responsibility is to impress his audience and not to let himself be amazed, as he cannot disclose his feelings and he has to adopt an impenetrable attitude, guided by the constant urge of staging his life, as a form of protection against the surrounding world, malevolent and rude.

\section{keywords}

dandyism, revolution, provocation, beauty, art

\section{mots-clés}

dandysme, révolution, provocation, beauté, art

\section{edyta kociubińska}

Enseignante-chercheuse en littérature française du XIXe siècle et maître de conférences HDR à I'Université Catholique de Lublin Jean-Paul II (Pologne), auteur de nombreuses études consacrées au naturalisme et à la décadence ; rédactrice en chef de la revue Quêtes littéraires ; correspondante polonaise de la Société des Études Romantiques et dixNeuviémistes ; membre associé du Centre Transdisciplinaire d'Épistémologie de la Littérature et des Arts vivants (Université Nice Sophia Antipolis). Actuellement, ses recherches portent sur le dandysme littéraire au XIXe siècle. 